\title{
Methylation and decreased expression of SHP-1 are related to disease progression in chronic myelogenous leukemia
}

\author{
YINGHUA LI ${ }^{1,2}$, LIN YANG $^{1}$, YUXIA PAN $^{1}$, JINGCI YANG $^{1}$, YINTAO SHANG $^{1}$ and JIANMIN LUO ${ }^{1}$ \\ ${ }^{1}$ Department of Hematology, The Second Hospital of Hebei Medical University, Key Laboratory of Hematology, Shijiazhuang, \\ Hebei 050000; ${ }^{2}$ Department of Hematology, Harrison International Peace Hospital, Hengshui, Hebei 053000, P.R. China
}

Received January 24, 2014; Accepted March 4, 2014

DOI: $10.3892 /$ or.2014.3098

\begin{abstract}
Despite the unprecedented success of tyrosine kinase inhibitors (TKIs) in treating chronic myelogenous leukemia (CML), some patients nevertheless progress to advanced stages of the disease. Thus far, the biological basis leading to $\mathrm{CML}$ progression remains poorly understood. SH2-containing tyrosine phosphatase 1 (SHP-1) is reported to bind to $\mathrm{p} 210^{\mathrm{BCR}-\mathrm{ABL} 1}$ and to function as a tumor suppressor. Furthermore, its substrates have been found to be essential for $\mathrm{p} 210^{\mathrm{BCR}-\mathrm{ABL} 1}$ leukemogenesis or CML progression. In the present study, we found that SHP-1 mRNA and protein levels were markedly decreased in patients in the accelerated and blastic phases of CML (AP-CML and BP-CML) compared to those in the chronic phase (CP-CML). In vitro, we demonstrated that overexpression of SHP-1 reduced p210 ${ }^{\mathrm{BCR}-\mathrm{ABL} 1}$ protein expression and activity in the K562 CML cell line and negatively regulated the AKT, MAPK, MYC and JAK2/STAT5 signaling pathways. Moreover, using a methylation-specific polymerase chain reaction (MSP) assay, abnormal methylation of the SHP-1 gene promoter region was found both in K562 cells and bone marrow (BM) or peripheral blood (PB) cells from AP-CML and BP-CML patients. In conclusion, our findings suggest that decreased expression levels of SHP-1 caused by aberrant promoter hypermethylation may play a key role in the progression of CML by dysregulating BCR-ABL1, AKT, MAPK, MYC and JAK2/STAT5 signaling.
\end{abstract}

Correspondence to: Professor Jianmin Luo, Department of Hematology, The Second Hospital of Hebei Medical University, Key Laboratory of Hematology, 215 West Heping Road, Shijiazhuang, Hebei 050000, P.R. China

E-mail: luojm2011@163.com

Abbreviations: CML, chronic myelogenous leukemia; $\mathrm{CP}$, chronic phase; AP, accelerated phase; BP, blastic phase; PBMCs, normal peripheral blood mononuclear cells; $\mathrm{U}$, amplified products used as primers for the unmethylated sequence; $\mathrm{M}$, amplified products used as primers for the methylated sequence; IM, imatinib mesylate; MSP, methylation-specific polymerase chain reaction

Key words: chronic myelogenous leukemia, SHP-1, blastic transformation, methylation

\section{Introduction}

Chronic myelogenous leukemia (CML) is a hematopoietic stem cell disease characterized by a reciprocal translocation between chromosomes 9 and 22, resulting in the formation of the Philadelphia $(\mathrm{Ph})$ chromosome. This translocation $(9 ; 22)$ results in the head-to-tail fusion of the breakpoint cluster region $(\mathrm{BCR})$ gene on chromosome 22 at band q11 with the Abelson murine leukemia (ABL) gene located on chromosome 9 at band q34. The product of the BCR-ABL1 fusion gene encodes a $210-\mathrm{kDa}$ oncoprotein designated as p210 ${ }^{\mathrm{BCR}-\mathrm{ABL} 1}$, which demonstrates constitutive tyrosine kinase activity $(1,2)$. p $210^{\text {BCR-ABL1 }}$ plays a key role in the pathogenesis of CML via its interaction with numerous molecules regulating cell survival, proliferation and differentiation (3). Typically, CML passes through three different clinicopathological phases: a chronic phase (CP), an accelerated phase (AP) and a blastic phase (BP) (4). Untreated chronic phase CML (CP-CML) patients eventually progress to advanced phases (AP and BP) within 3-5 years (5). Tyrosine kinase inhibitor (TKI) resistance or blastic transformation has been found in $\sim 15-20 \%$ of patients with CML, although the molecular mechanisms remain poorly understood $(3,6-8)$. However, recent studies have demonstrated that the bulk of the genetic changes associated with progression occur in the transformation from $\mathrm{CP}$ to AP or BP (9).

SHP-1 or PTPN6 (also previously referred to as SH-PTP1, $\mathrm{PTP} 1 \mathrm{C}, \mathrm{HCP}$ or Hcph) is a non-receptor protein tyrosine phosphatase (PTP) that is predominantly expressed in hematopoietic cells and plays an important role in the negative regulation of growth-promoting signaling molecules, such as JAKs/STATs and PI3K/AKT/mTOR (10-12). Mice deficient in SHP-1 (SHP-1 ${ }^{-/}$and SHP-1 ${ }^{+-}$; motheaten and viable motheaten mice, respectively) demonstrate marked myeloid proliferation $(13,14)$. Moreover, SHP-1 promoter methylation causes loss of SHP-1 expression in hematological malignancies, resulting in the activation of the JAK/STAT pathway (15-17). SHP-1 also accounts for resistance to imatinib (IM) treatment in patients with CML (18), as it appears to be physically associated with BCR-ABL1 and is able to block BCR-ABL-dependent and -independent signaling pathways $(19,20)$. In the present study, we demonstrated that: i) the expression levels of SHP-1 are markedly decreased in patients with BP-CML or AP-CML compared with CP-CML; ii) SHP-1 reduces $\mathrm{p} 210^{\mathrm{BCR}-\mathrm{ABL} 1}$ 
protein expression and activity and negatively regulates the AKT, MAPK, MYC and JAK2/STAT5 signaling pathways; and iii) abnormal methylation of the SHP-1 promoter region occurs in K562 cells and cells from patients with advanced CML. Therefore, low levels of SHP-1 caused by aberrant promoter hypermethylation may be related to CML blastic transformation by dysregulating the BCR-ABL1, AKT, MAPK, MYC and JAK2/STAT5 signaling pathways.

\section{Materials and methods}

Patients and healthy donors. Between December 2010 and June 2013, bone marrow (BM) aspirates or peripheral blood (PB) cells were collected from 94 consecutive patients with CML treated at the Second Hospital, Hebei Medical University and 11 healthy donors under an approved institutional protocol. The definitions of CP, AP and BP corresponded to those defined in the European Leukemia Net (ELN). The present study was approved by the Medical Ethics Committee of the Second Hospital of Hebei Medical University.

Tumor cell line. The BCR-ABL-positive K562 cell line (maintained in our laboratory) was maintained in RPMI-1640 medium supplemented with $10 \%$ fetal bovine serum (FBS) in a humidified $5 \% \mathrm{CO}_{2}$ atmosphere at $37^{\circ} \mathrm{C}$. The cells were passed or the medium was renewed every 2 to 3 days, and the cells were prepared for experimental procedures when they reached log-phase growth and $80 \%$ confluency.

Reagents and antibodies. Imatinib mesylate (IM) and nilotinib were kindly provided by Novartis Pharma Stein AG (Basel, Switzerland). Puromycin 2HCL was purchased from Beijing Solarbio (Beijing, China). SHP-1, Crk-L and GAPDH antibodies were obtained from Santa Cruz Biotechnology (Santa Cruz, CA, USA); JAK2 and phospho-JAK2 (pTyr1007/1008) antibodies were obtained from Abcam Inc., (Cambridge, UK); STAT5, phospho-STAT5 (pTyr694), AKT, phospho-AKT (pSer473), MAPK and phospho-MAPK p44/42 (Erk1/2) (Thr202/Tyr204) antibodies were purchased from Cell Signaling Technology (Danvers, MA, USA); Alexa Fluor ${ }^{\circledR}$ 488 mouse anti-CrkL (pY207), the isotype control antibody, anti-normal-rabbit immunoglobulins were obtained from BD Biosciences (San Diego, CA, USA).

Assessment of apoptosis. The rate of apoptosis was evaluated using an Annexin V/PI apoptosis kit (MultiSciences Biotech Co., Ltd., Hangzhou, China) according to the manufacturer's instructions. For each sample, $1-5 \times 10^{5}$ cells were analyzed on a BD FACSCalibur (Becton-Dickinson, San Jose, CA, USA). Annexin V/PI discriminates intact cells (Annexin V-/PI') from early apoptotic cells (Annexin $\mathrm{V}^{+} / \mathrm{PI}^{-}$) and late apoptotic/ necrotic cells (Annexin $\mathrm{V}^{+} / \mathrm{PI}^{+}$).

Cell cycle analysis. One million cells were centrifuged at $1,000 \times \mathrm{g}$ for $3 \mathrm{~min}$, washed twice with ice-cold PBS, resuspended in $70 \%$ ice-cold ethanol and fixed at $4{ }^{\circ} \mathrm{C}$ overnight or stored at $-20^{\circ} \mathrm{C}$. The cells were collected by centrifugation and washed twice with precooled PBS to remove the ethanol. PI (10 $\mu \mathrm{l}$ ) was then added into $500 \mu \mathrm{l}$ of binding buffer, and the cells were incubated at $4^{\circ} \mathrm{C}$ in the dark for $30 \mathrm{~min}$. Samples were analyzed on a FACSCalibur (Becton-Dickinson) using ModFit Lt3.0 software (Verity Software House).

Cell line viability and proliferation assays. Cell line viability and proliferation were assessed using a Cell Counting Kit-8 CCK-8/WST-8 kit (Dojindo Laboratories, Kumamoto, Japan), respectively, according to the manufacturer's instructions.

Immunoblotting. Cells $\left(10^{7}\right)$ were harvested, washed twice with ice-cold PBS and lysed in $100 \mu \mathrm{l}$ of SDS lysis buffer [50 mM DTT, 2\% SDS, $62.5 \mathrm{mM}$ Tris (pH 6.8), 10\% glycerin]. Lysates were centrifuged $\left(12,000 \times \mathrm{g}, 15 \mathrm{~min}, 4^{\circ} \mathrm{C}\right)$, denatured $\left(10 \mathrm{~min}, 100^{\circ} \mathrm{C}\right)$, subjected to $8-12 \%$ SDS-PAGE and electrotransferred to polyvinylidene fluoride (PVDF) membranes (0.45 $\mu \mathrm{m}$; Millipore, Billerica, MA, USA). The membranes were blocked in $10 \%$ bovine serum albumin (BSA) at $4^{\circ} \mathrm{C}$ overnight or at room temperature for $2 \mathrm{~h}$ and immunoblotted with antibodies (1:1,000 dilution) at room temperature for $40 \mathrm{~min}$. The membranes were then incubated with horseradish peroxidase (HRP)-labeled immunoglobulin (Santa Cruz Biotechnology) at room temperature for $30 \mathrm{~min}$ at a 1:2,000 dilution followed by reaction using an enhanced chemiluminescence (ECL) kit (Amersham Pharmacia Biotech Inc., Piscataway, NJ, USA). The membranes were exposed to X-ray film (Kodak, Rochester, NY USA), and protein expression was expressed as densitometric units after normalization to GAPDH levels (ImageJ).

SYBR-Green-based qRT-PCR. Mononuclear cells were isolated from the BM aspirates or peripheral blood by centrifugation on a Ficoll-Hypaque gradient. Total RNA was extracted using the TRIzol reagent (Invitrogen-Life Technologies, Carlsbad, CA, USA) according to the manufacturer's instructions. Total RNA $(1 \mu \mathrm{g})$ was reverse transcribed in $20 \mu \mathrm{l}$ using an All-in-One First-Strand cDNA Synthesis kit (GeneCopoeia Inc., Guangzhou, China), and cDNA (1 $\mu \mathrm{l})$ was amplified using a SYBR-Green-based quantitative PCR (qPCR) reaction using an All-in-One ${ }^{\mathrm{TM}}$ qPCR Mix kit (GeneCopoeia Inc.). The qPCR conditions consisted of 35 cycles at $94^{\circ} \mathrm{C}$ for $10 \mathrm{sec}$, $65^{\circ} \mathrm{C}$ for $45 \mathrm{sec}$ and $72^{\circ} \mathrm{C}$ for $10 \mathrm{sec}$. The primer sequences are documented in Table I. The qPCR reactions were performed in triplicate using a Three-Step-with-Melt PCR system. $\beta$-actin was monitored as the housekeeping control gene for equal amplification. SHP-1 mRNA was normalized to $\beta$-actin levels to obtain the relative threshold cycle $(\Delta \mathrm{Ct})$, and the resulting number was then related to the $\Delta \mathrm{Ct}$ of the control gene to obtain the relative expression level $\left(2^{-\Delta \Delta \mathrm{Ct}}\right)$.

CrkL phosphorylation assay. Phosphorylated CrkL (pCrkL) analysis by flow cytometry was performed as previously described $(21,22)$. One million cells were resuspended in $500 \mu \mathrm{l}$ of $2 \%$ paraformaldehyde and fixed for $10 \mathrm{~min}$ at $37^{\circ} \mathrm{C}$, then chilled on ice for $1 \mathrm{~min}$. The cells were harvested by centrifugation (770 x g, $3 \mathrm{~min}$ ), and $500 \mu \mathrm{l}$ of $90 \%$ methanol was added. The cells were vortexed briefly and incubated on ice for $30 \mathrm{~min}$. The cells were then washed (throughout with $1 \mathrm{ml}$ of incubation buffer containing phosphate-buffered saline and $0.5 \%$ bovine serum albumin), harvested and resuspended in $25 \mu \mathrm{l}$ of incubation buffer and incubated at room temperature for 10 min. Alexa Fluor ${ }^{\circledR} 488$ mouse anti-CrkL (pY207) was 
Table I. MSP and SYBR-Green-based qRT-PCR: primer sequences and products.

\begin{tabular}{llll}
\hline Gene & Sense primer (5'-3') & Antisense primer (5'-3') & Products (bp) \\
\hline$\beta$-actin & gagctacgagctgcctgac & ggtagtttcgtggatgccacag & 121 \\
SHP-1 & aacagccgtgtcatcgtcat & atcaggtctccattgtccagc & 191 \\
SHP-1M-MSP & gaacgttattatagtatagcgttc & tcacgcatacgaacccaaacg & 158 \\
SHP-1U-MSP & tcacgcatacgaacccaaacg & ttcacacatacaaacccaaacaat & 158 \\
\hline
\end{tabular}

$\mathrm{U}$, amplified products used as primers for the unmethylated sequence. M, amplified products used as primers for the methylated sequence. MSP, methylation-specific polymerase chain reaction.

Table II. Characteristics of the patients included in the study.

\begin{tabular}{|c|c|c|c|c|}
\hline & CP-CML $(n=42)$ & AP-CML $(n=22)$ & BP-CML $(n=30)$ & P-value ${ }^{a}$ \\
\hline Age (years), median (range) & $45(16-72)$ & $45(33-69)$ & $50(8-68)$ & 0.37 \\
\hline Male/female, $(\mathrm{n} / \mathrm{n})$ & $24 / 18$ & $14 / 8$ & $16 / 14$ & 0.87 \\
\hline WBCs, $\times 10^{9} / 1$, median (range) & $199(33-427)$ & $199(60-373)$ & $236(60-356)$ & 0.48 \\
\hline Hemoglobin level (g/dl) & $95(62-138)$ & $88(68-138)$ & $89(62-117)$ & 0.32 \\
\hline PLT count $\times 10^{9} / 1$, median (range) & $470(39-998)$ & $350(87-1147)$ & $308(54-1208)$ & 0.10 \\
\hline
\end{tabular}

WBCs, white blood cells. PLT, platelet. ${ }^{\text {P }}$-value, comparison among the 3 groups.

added $(3 \mu \mathrm{l})$, and the cells were incubated at room temperature in the dark for $30 \mathrm{~min}$. The cells were washed twice and then analyzed by flow cytometry (Becton-Dickinson) using CellQuest Pro Software (Becton-Dickinson) for data analysis. The amount of pCrkL in the sample was determined as the geometric mean fluorescence intensity (MFI) minus the MFI value of the isotype control.

Methylation-specific polymerase chain reaction (MSP). Genomic DNA was isolated, sodium bisulfite-modified and purified with the EZ DNA Methylation-Direct ${ }^{\mathrm{TM}}$ kit (Zymo Research, Irvine, CA, USA) according to the manufacturer's protocol. Primers (Table I) were designed to detect the methylated and unmethylated sequence of the promoter region for exon $1 \mathrm{~b}$ of the SHP-1 gene (15). Initial denaturation at $94^{\circ} \mathrm{C}$ for 3 min was followed by 40 cycles of denaturation at $94^{\circ} \mathrm{C}$ for $30 \mathrm{sec}$, an annealing step at $55^{\circ} \mathrm{C}$ for $1 \mathrm{~min}$, an extension step at $72^{\circ} \mathrm{C}$ for $1 \mathrm{~min}$ and a final extension step at $72^{\circ} \mathrm{C}$ for $10 \mathrm{~min}$. The products were separated by electrophoresis on $2 \%$ agarose gels.

Tumor cell line transfection. The lentiviral expression plasmids, pEX-SHP-1-puro-Lv105 and pEX-EGFP-puro-Lv105, were constructed and packaged by GeneCopoeia Inc. pEXSHP-1-puro-Lv105 contains the open reading frame (ORF) of human SHP-1 (National Center for Biotechnology Information NM_002831.4) under the control of the CMV promoter. K562 cells were infected with the specified vector and the mock vectors according to the manufacturer's protocol.

Statistical analysis. Data values represent the mean \pm SD of at least 3 independent experiments. Data were analyzed statistically using SAS 9.1.3 (SAS Institute Inc., Cary, NC, USA; 5695502). Data were tested for normal distribution. One-way ANOVA analysis (LSD), independent-samples t-test, non-parametric Mann-Whitney test, Bonferroni-correction non-parametric test, RC contingency table Chi-square test, or Fisher's exact test were applied; P-values $<0.05$ were considered to indicate statistically significant results.

\section{Results}

Decreased expression of SHP-1 is associated with progression of $C M L$. The mRNA levels of SHP-1 in BM aspirates or peripheral blood mononuclear cells (PBMCs) from 94 (CP, 42; AP, 22; BP, 30) highly heterogeneous CML patients were assessed using SYBR Green-based relative quantitative RT-PCR. The main clinical and hematologic features of the 3 groups of patients are summarized in Table II, and there were no significant differences in the main features among the groups. SHP-1 mRNA transcripts were significantly lower $(\mathrm{P}<0.01)$ in the samples from the AP and BP patients $(0.79 \pm 0.36$ and $0.78 \pm 0.40$, respectively) compared to those from the $\mathrm{CP}$ patients $(1.18 \pm 0.64)$. We observed no significant difference in SHP-1 mRNA levels between AP-CML and BP-CML patients $(\mathrm{P}=0.987)$ (Fig. 1A).

We detected the expression levels of the SHP-1 protein in $\mathrm{BM}$ aspirate mononuclear cells from 10 cases each in three different stages of CML using immunoblotting assays; similarly, the results showed that the levels of SHP-1 protein were lower in AP-CML $(0.59 \pm 0.07)$ and BP-CML $(0.59 \pm 0.07)$ patient-derived mononuclear $\mathrm{BM}$ cells compared to $\mathrm{CP}-\mathrm{CML}$ $(1.02 \pm 0.13)(\mathrm{P}<0.05)$ (Fig. 1B). 
A

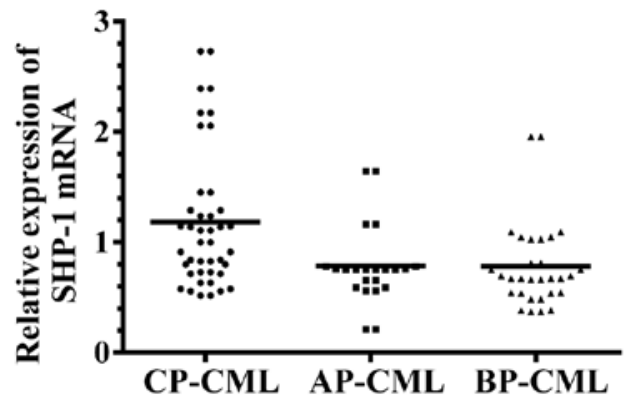

B

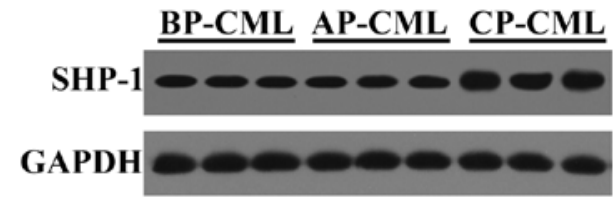

C

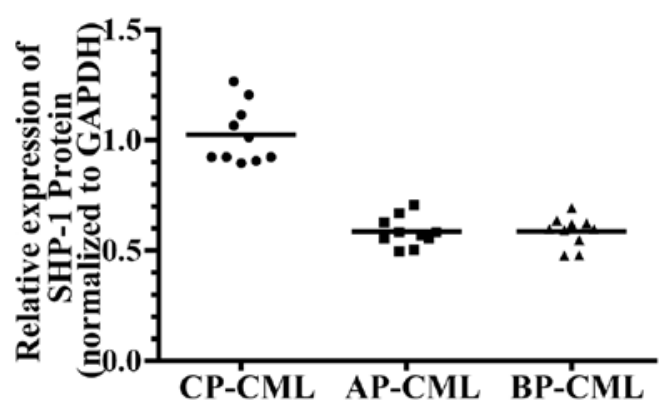

Figure 1. Expression of SHP-1 mRNA and protein in BM or PB samples from patients in different phases of CML. (A) Levels of SHP-1 mRNA as assessed by qRT-PCR. Straight lines indicate the relative expression levels of SHP-1 in the various groups. Statistical analysis was performed using Bonferronicorrection non-parametric test, which showed a significant difference in SHP-1 expression in the AP-CML and BP-CML patients compared to the CP-CML patients $(\mathrm{P}<0.05)$. There was no difference in SHP-1 mRNA levels between the AP-CML and BP-CML patients. (B) SHP-1 protein levels in BM or PB cells from CML patients (top panel). SHP-1 protein expression was determined by immunoblotting with GAPDH as a control (bottom panel). (C) Scatter plots of the relative density of SHP-1 protein normalized to GAPDH in $\mathrm{BM}$ cells from the AP-CML, BP-CML and CP-CML patients. The expression levels of SHP-1 protein were lower in the AP-CML and BP-CML compared to the CP-CML patient-derived mononuclear $\mathrm{BM}$ cells $(\mathrm{P}<0.05)$.

Effects of SHP-1 on the biological characteristics of K562 cells. To assess the effects of SHP-1 on K562 cells, we stably expressed SHP-1 in K562 cells and puromycin-selected $\mathrm{K} 562^{\mathrm{EGFP}}$ and $\mathrm{K} 562^{\mathrm{SHP}-1}$ cells. After transfection, SHP-1 protein was re-expressed in the K562 $2^{\text {SHP-1 }}$ cells. CCK-8 assays and cell counts showed that the cell proliferation rate was sharply reduced in the $\mathrm{K} 562^{\mathrm{SHP}-1}$ cells compared to the $\mathrm{K} 562^{\mathrm{EGFP}}$ control cells from the third day of culture (Fig. 2A).

We also observed a sharp increase in early and late stage apoptosis among the $\mathrm{K} 562^{\text {SHP-1 }}$ cells, as measured by Annexin V/ PI staining and flow cytometry, and the percentage of apoptotic K562 $2^{\text {SHP-1 }}$ cells was significantly higher than that of apoptotic K562 $2^{\text {EGFP }}$ control cells from the third day of culture (Fig. 2B).

Notably, an accumulation of cells in the G0/G1 phase and a reduction of cells in the $\mathrm{S}$ phase were observed from the third day of culture for the K562 $2^{\mathrm{SHP}-1}$ and $\mathrm{K} 562^{\mathrm{EGFP}}$ cells, whereas no difference was found in $\mathrm{G} 2 / \mathrm{M}$ cell populations for K562 $2^{\mathrm{SHP}-1}$ compared to K562 ${ }^{\mathrm{EGFP}}$ cells (Fig. 2C).
Overexpression of SHP-1 in K562 cells reduces p210 ${ }^{B C R-A B L I}$ expression and activity. To establish the effects of SHP-1 on BCR-ABL1, we first detected the expression of BCR-ABL1 in $\mathrm{K} 562^{\text {SHP-1 }}$ and $\mathrm{K} 562^{\mathrm{EGFP}}$ cells $72 \mathrm{~h}$ after transfection with pEXSHP-1-puro-Lv105. We found that SHP-1 controlled BCR-ABL1 protein levels, as SHP-1 overexpression caused a slight decrease in p210 ${ }^{\text {BCR-ABL1 }}$ protein in $\mathrm{K} 562^{\text {SHP-1 }}$ cells $(0.78 \pm 0.15)$ compared to the mock control K562 ${ }^{\mathrm{EGFP}}$ cells $(1.27 \pm 0.24)(\mathrm{P}=0.038)$ (Fig. 3A). However, the levels of BCR-ABL1 mRNA were not affected in the K562 ${ }^{\mathrm{SHP}-1}$ cells $(1.32 \pm 0.34)$ compared to the K562 ${ }^{\mathrm{EGFP}}$ cells $(1.18 \pm 0.20)(\mathrm{P}=0.64)$ (Fig. 3B).

The CrkL protein is a downstream signaling substrate of p210 ${ }^{\text {BCR-ABLI }}$, and the tyrosine phosphorylation of CrkL (pCrkL) has been identified as a surrogate marker of $\mathrm{p} 210^{\mathrm{BCR}-\mathrm{ABL} 1}$ tyrosine kinase activity in CML cells (21). We, therefore, examined the effects of SHP-1 on p210 ${ }^{\text {BCR-ABL1 }}$ activity by measuring the level of pCrkL by flow cytometry. The results showed that $\mathrm{pCrkL}$ was decreased by $63.1 \%$ following transfection of SHP-1 into K562 cells $(\mathrm{P}<0.01)$. Since previous research has demonstrated that treatment of K562 cells with IM results in a dose-dependent decrease in pCrkL (21), we also examined the effect of IM treatment $(50 \mathrm{nM})$ for $8 \mathrm{~h}$ on CrkL phosphorylation in $\mathrm{K} 562^{\mathrm{EGFP}}$ and $\mathrm{K} 562^{\mathrm{SHP}-1}$ cells. The results revealed a significant decrease in pCrkL between $\mathrm{K} 562^{\mathrm{SHP}-1}$ and $\mathrm{K} 562^{\mathrm{EGFP}}$ cells $(\mathrm{P}<0.01)$ (decreased by 88.2 and $60.3 \%$, respectively) following treatment with IM (50 nM) for $8 \mathrm{~h}$ (Fig. 3C).

Effects of SHP-1 on BCR-ABL1-independent signaling pathways in K562 cells. The ability of $\mathrm{p} 210^{\mathrm{BCR}-\mathrm{ABL} 1}$ to induce and sustain CML depends on the modulation of the expression and/ or activity of downstream signaling molecules, such as those involved in the RAS/MAPK, PI3K/AKT, MYC and JAK/ STAT pathways. Thus, we investigated whether SHP-1 affects the expression and/or activity of these molecules controlling cell growth, survival and differentiation.

At $48 \mathrm{~h}$ after transfection with pEX-SHP-1-puro-Lv105, the transfected cells were collected, and western blotting was performed using various specific antibodies. Expression was estimated by dividing the intensity of the bands normalized to the GAPDH loading controls by the normalized intensity of the bands from K562 $2^{\mathrm{EGFP}}$ control cells. Overexpression of SHP-1 resulted in a marked decrease in MYC and the phosphorylated forms of JAK2, STAT5, AKT and MAPK $(\mathrm{P}<0.01)$; however, the unphosphorylated forms of these molecules were not significantly affected ( $>>0.05)$ (Fig. 4). These results indicate that AKT, MAPK, MYC and JAK2/STAT5 are downstream of SHP-1 and that SHP-1 negatively regulates AKT, MAPK, Myc and JAK2/STAT5 signaling.

To further investigate the SHP-1-mediated negative regulation of these molecules using a BCR-ABL1-independent model, we stably expressed SHP-1 in K562 cells and treated puromycin-selected $\mathrm{K} 562^{\mathrm{EGFP}}$ and $\mathrm{K} 562^{\mathrm{SHP}-1}$ cells with $50 \mathrm{nM}$ IM for $8 \mathrm{~h}$. The results revealed a decrease in phosphorylated AKT, MAPK and JAK2/STAT5 in K562 ${ }^{\text {SHP-1 }}$ cells in comparison to $\mathrm{K} 562^{\mathrm{EGFP}}$ cells $(\mathrm{P}<0.01)$ (Fig. 4).

Decreased expression of SHP-1 due to aberrant CpG methylation of the SHP-1 promoter in both K562 cells and cells from patients with advanced CML. To assess the function of SHP-1 in K562 cells, we initially assessed SHP-1 mRNA 
A

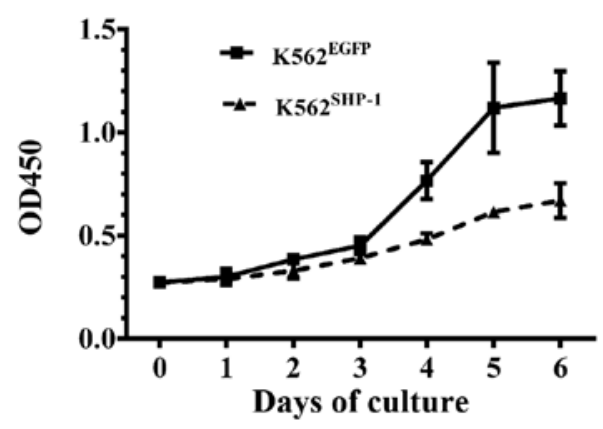

B

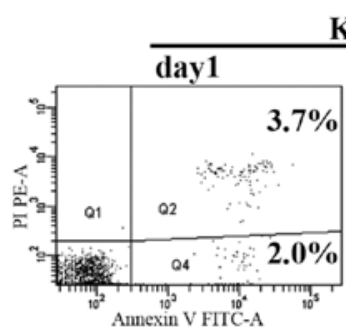

K562 ${ }^{\text {EGFP }}$

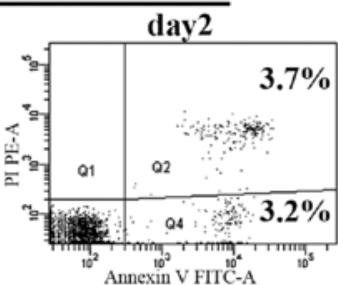

day3

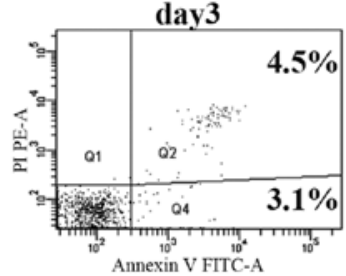

day5
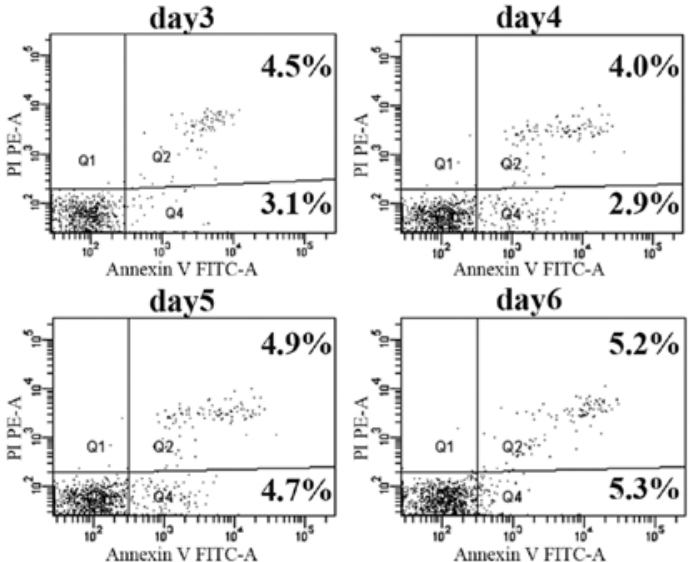

day6

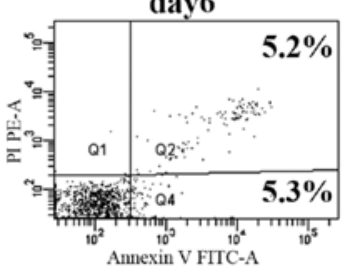

C

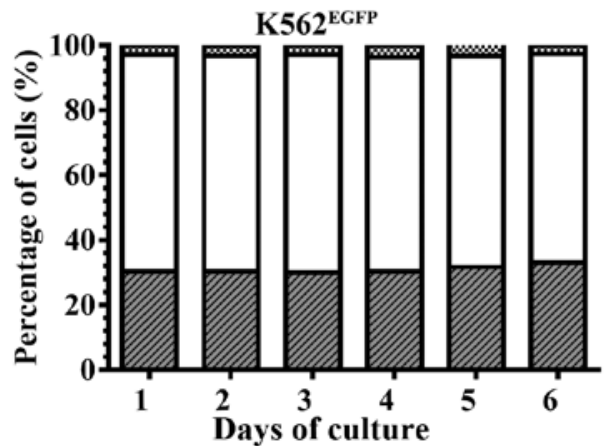

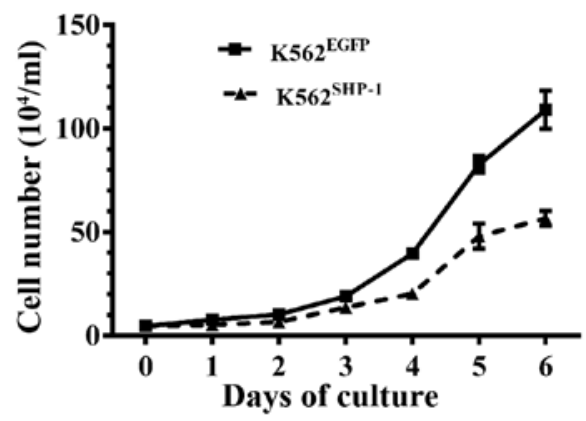

$\mathrm{K562}^{\text {SHP-1 }}$
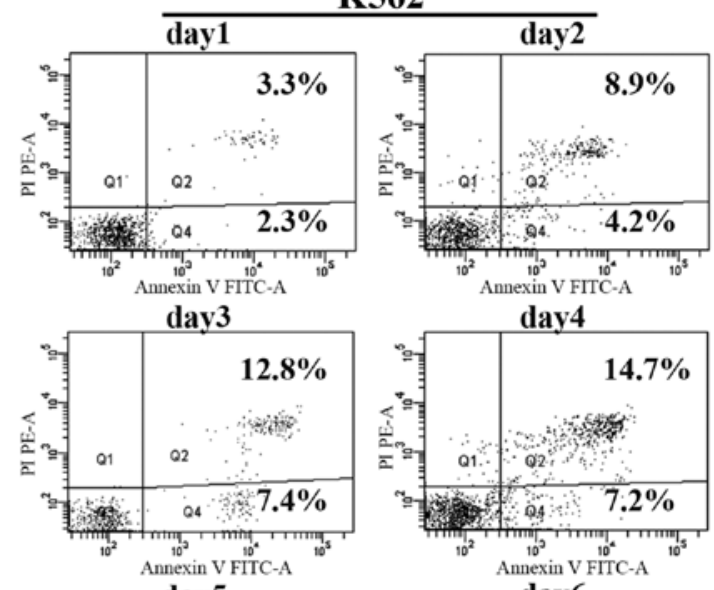

day5

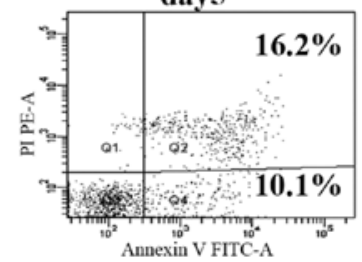

day6
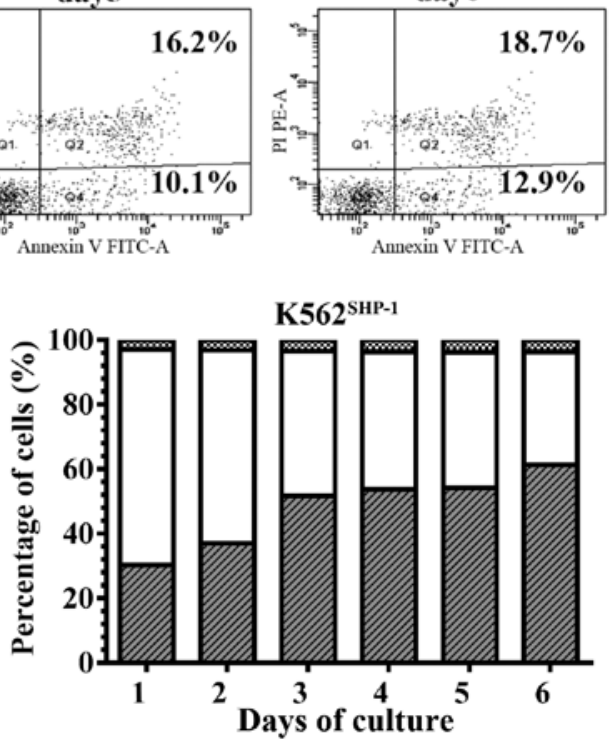

Figure 2. Effects of SHP-1 on the biological characteristics of K562 cells. (A) Antiproliferative effect of SHP-1 on K562 cells at different time points. Cell viability was determined by CCK-8 assays (left) and cell counts (right) after transfection of SHP-1. The proliferation rate was sharply reduced in the K562 ${ }^{\text {SHP-1 }}$ cells compared to the K562 ${ }^{\text {EGFP }}$ cells from the third day of culture. (B) The apoptotic rate detected using flow cytometry. The percentage of apoptotic K562 ${ }^{\text {SHP-1 }}$ cells was significantly higher than that of apoptotic K562 ${ }^{\mathrm{EGFP}}$ control cells from the third day of culture. (C) Overexpression of SHP-1 arrested the K562 cells in the G0/G1 phase. An accumulation of cells in the G0/G1 phase and a reduction of cells in the S phase were observed from the third day of culture for K562 ${ }^{\text {SHP-1 }}$ and K562 ${ }^{\mathrm{EGFP}}$ cells.

levels in K562 cells compared to PBMCs from healthy donors. qRT-PCR analysis indicated that SHP-1 mRNA was significantly reduced by $\sim 98 \%$ in K562 cells $(0.03 \pm 0.01)$ compared to normal PBMCs $(1.23 \pm 0.53)(\mathrm{P}=0.005)$, as previously reported (Fig. 5A). Consistent with these results, SHP-1 protein was expressed in PBMCs from healthy donors but was not detected in the K562 cells by western blot analysis. In addition, SHP-1 was not detected after treatment with IM (200 nM for $72 \mathrm{~h}$ ) or nilotinib (150 nM for $72 \mathrm{~h}$ ) (Fig. 5B).
We also analyzed whether epigenetic modifications could explain the decrease in SHP-1 levels observed in cells from patients with advanced CML. Genomic DNA obtained from primary BM samples or PBMCs from 94 CML patients (Table II) was analyzed using MSP. The frequency of SHP-1 DNA promoter methylation at selected loci in $42 \mathrm{CP}-\mathrm{CML}$ samples was $23.8 \%(n=10)$, and methylated regions were detected in all advanced CML samples with lower SHP-1 expression ( $\mathrm{P}=0.001)$ (Fig. 5C). 
A
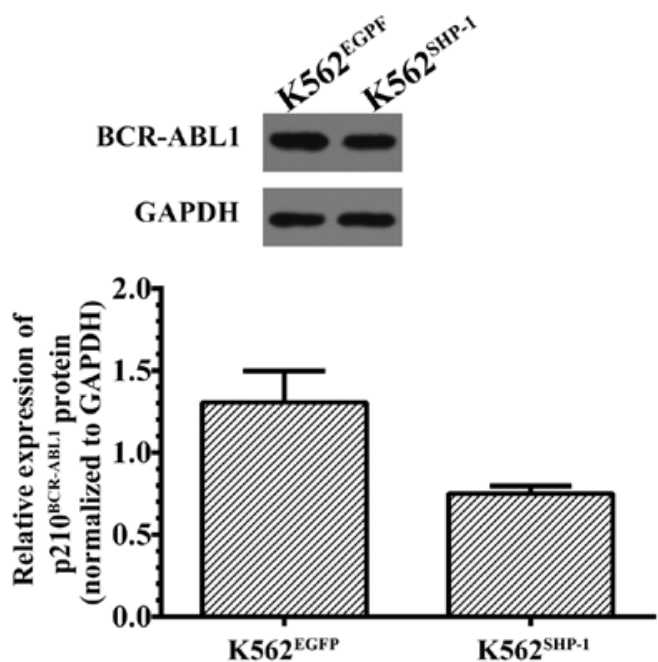

B

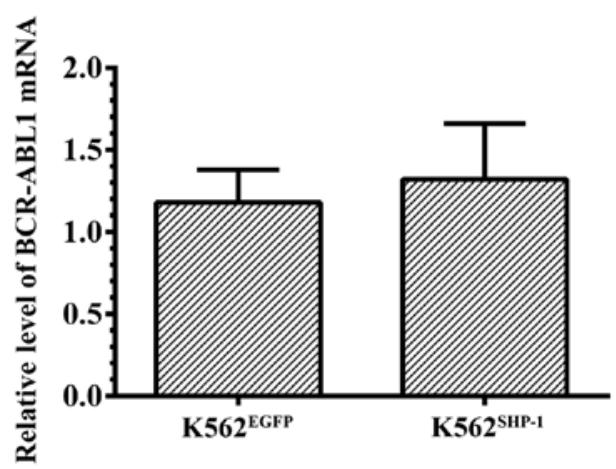

C

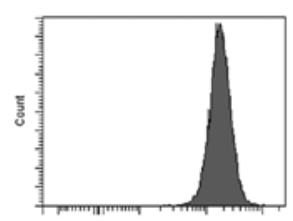

FL FTC FTOA

K562 ${ }^{\text {EGFP }}$

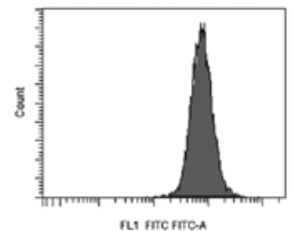

IM+K562 EGFP

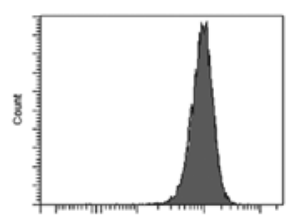

FLI FITCFITCA K562 ${ }^{\text {SHP-1 }}$

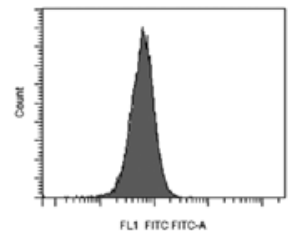

$\mathrm{IM}+\mathrm{K562}^{\mathrm{SHP}-1}$

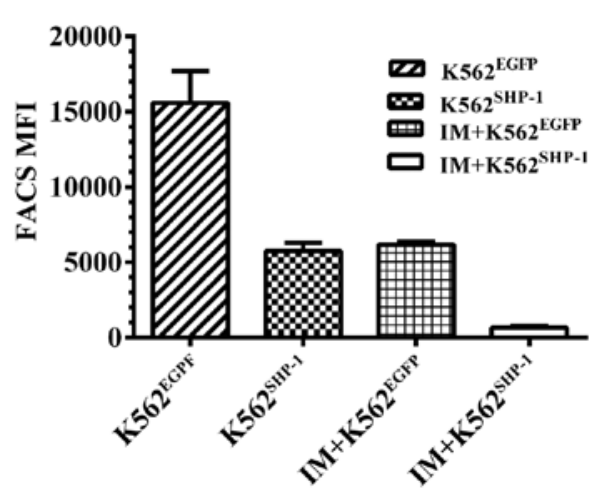

Figure 3. Effects of SHP-1 on p210 ${ }^{\text {BCR-ABL1 }}$ expression and activity. (A) SHP-1 mildly decreased the expression of p210 ${ }^{\text {BCR-ABL1 }}$ protein in the K562 ${ }^{\text {SHP-1 }}$ cells compared to the K562 ${ }^{\mathrm{EGFP}}$ cells, $\mathrm{P}<0.05$. (B) Overexpression of SHP-1 did not affect the levels of BCR-ABL1 mRNA in the K562 $2^{\mathrm{SHP}-1}$ cells compared to the $\mathrm{K} 562^{\text {EGFP }}$ cells $(\mathrm{P}>0.05)$. (C) pCrkL MFI in K562 ${ }^{\text {EGFP }}$ and K562 ${ }^{\text {SHP-1 }}$ cells. pCrkL MFI was decreased in the K562 $2^{\text {SHP-1 }}$ cells compared to the K562 ${ }^{\text {EGFP }}$ cells either withour or with treatment of $50 \mathrm{nM}$ IM for $8 \mathrm{~h}(\mathrm{P}<0.05)$.

A

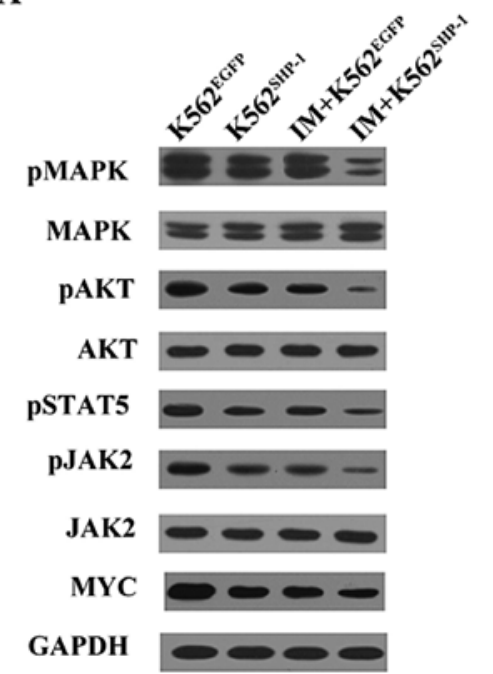

B

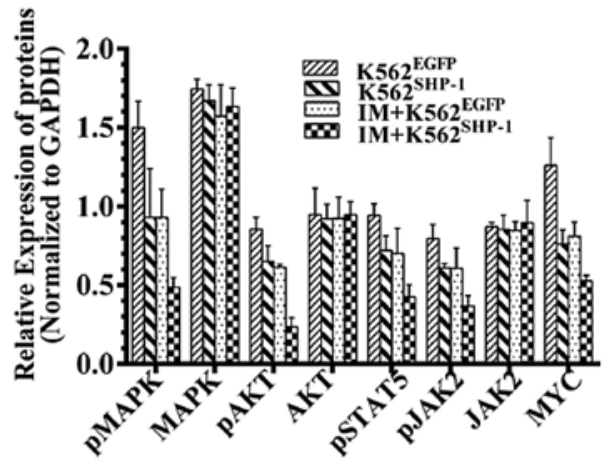

Figure 4. Effects of SHP-1 on BCR-ABL1-independent signaling pathways in K562 cells. (A) Signaling pathway analysis by western blot analysis in K562 ${ }^{\text {EGFP }}$ and $\mathrm{K} 562^{\mathrm{SHP}-1}$ cells prior to and after exposure to $50 \mathrm{nM}$ IM for $8 \mathrm{~h}$. (B) Densitometric analysis after normalization to GAPDH. MYC and pJAK2, pSTAT5, pAKT and pMAPK were decreased in the K562 ${ }^{\text {SHP-1 }}$ cells compared to the K562 ${ }^{\text {EGFP }}$ cells withour or with treatment of $50 \mathrm{nM} \mathrm{IM}$ for $8 \mathrm{~h}$ (P<0.05).

Previous studies in malignant lymphoma and multiple myeloma have implicated SHP-1 gene methylation as responsible for the marked decrease in SHP-1 levels. Our MSP analysis revealed that the SHP-1 promoter was unmethylated in all 11 normal human samples but was methylated in K562 cells. As TKIs have become first-line therapy in CML, we 
A

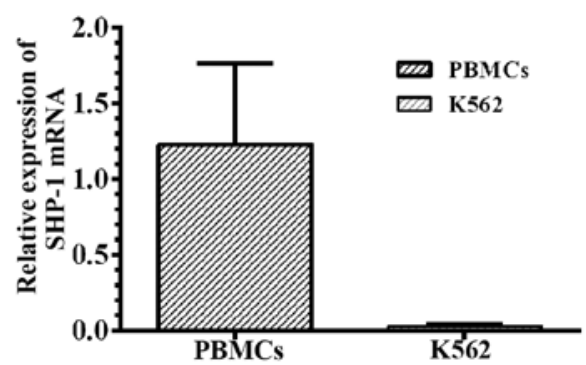

B

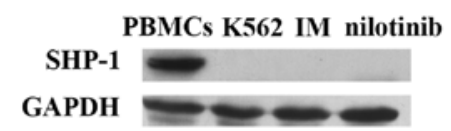

C

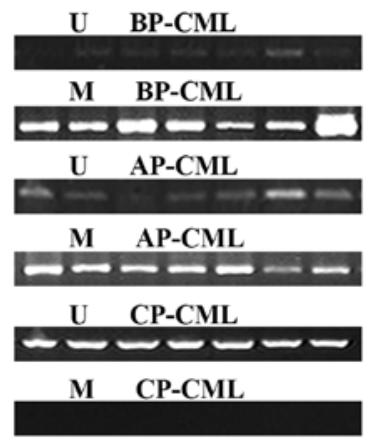

D

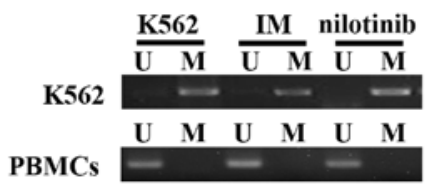

Figure 5. CpG methylation of the SHP-1 promoter in K562 cells and in cells from patients with advanced CML. (A) mRNA levels in K562 cells and PBMCs. (B) Expression of SHP-1 protein in K562 cells and normal PBMCs, and the effect of IM and nilotinib on SHP-1 protein expression in K562 cells. (C) Methylation analysis in patients with CML by MSP. (D) Methylation status in PBMCs and K562 cells, and the effect of IM (200 nM for $72 \mathrm{~h}$ ) and nilotinib (150 $\mathrm{nM}$ for $72 \mathrm{~h}$ ) on methylation of the SHP-1 promoter in K562 cells.

also investigated whether the levels of SHP-1 were affected by TKI treatment. However, treatment with IM $(200 \mathrm{nM}$ for $72 \mathrm{~h}$ ) or nilotinib ( $150 \mathrm{nM}$ for $72 \mathrm{~h}$ ) did not modify the methylation status of SHP-1, and no SHP-1 protein was detected (Fig. 5D).

\section{Discussion}

CML accounts for $15 \%$ of adult leukemias. The median age of disease onset is 50-60 years, although CML can occur in all age groups (23). The oncogenic potential of $\mathrm{p} 210^{\text {BCR-ABL1 }}$ has been demonstrated by the ability of this protein to transform hematopoietic progenitor cells both in vitro and in vivo. The p210 ${ }^{\text {BCR-ABL1 }}$ protein becomes constitutively active as a protein tyrosine kinase and increases proliferation, affects differentiation and blocks apoptosis. CML occurs in 3 distinct phases (CP, AP and BP). Before the advent of BCR-ABL1 TKIs, all patients with CP-CML progressed spontaneously to the advanced phases of CML, and patients with BP-CML demonstrate a median survival time of approximately 6 months.
Some patients progress directly to BP without an intermediate stage. Although TKI therapy alone or in combination with conventional chemotherapy followed by allogeneic hematopoietic stem cell transplantation has improved prognosis to some degree, these responses are not durable in BP-CML (24). Currently, the biological basis of BP is poorly understood, and reliable predictive markers that can identify those at risk of progression from CP-CML to advanced phases of CML are lacking. Gene expression profiling has shown a close correlation in the gene expression profile between CP-CML and advanced-phase CML (9). In the present study, we found that the level of the SHP-1 phosphatase was significantly decreased in cells from patients with advanced-phase CML as well as K562 cells, and this reduction in SHP-1 levels was related to the methylation status of the SHP-1 promoter.

The SHP-1 phosphatase is considered a negative regulator of cell proliferation and differentiation through its activity in dephosphorylating growth factors and cytokine receptors, including JAK/STAT and PI3K-AKT signaling components $(25,26)$. Indeed, constitutive activation of the JAK/STAT pathway contributes to the development and progression of CML (27-31). SHP-1 expression has also been shown to be important for K562 differentiation in response to various differentiating inducers (19). Thus, we suggest that loss of SHP-1 function may play a key role in the progression to blast crisis in CML. Indeed, as our previous research and studies by others have shown that SHP-1 gene mutation is infrequent in acute leukemia and mutations are not observed in CML cell lines or clinical samples $(32,33)$, it is tempting to speculate that epigenetic abnormality of the SHP-1 gene may contribute to blastic transformation in CML. Previously, decreased expression of SHP-1 in the progression of CML was reported in a study that assessed expression in the BM from 30 patients with CML using RT-PCR and immunohistochemistry (33). In the present study, we evaluated the expression of SHP-1 mRNA and protein using SYBR-Green-based qRT-PCR and western blot methods, respectively, in 94 patients with CML at different stages and explored the possible role of SHP-1 in CML progression.

When we examined BM or PB samples from patients with CML, we found that SHP-1 mRNA levels were significantly decreased in AP and BP samples compared to CP samples $(0.78 \pm 0.40$ and $0.79 \pm 0.36$ in AP and $\mathrm{BP}$, respectively, vs. $1.18 \pm 0.64$ in $\mathrm{CP}$ ), which is similar to the results of a previous study (33). Furthermore, the expression of SHP-1 protein was lower in advanced CML compared to CP-CML. These findings suggest that with CML progression, the levels of SHP-1 mRNA and protein are markedly decreased. In agreement with our observations in the advanced stages of CML, previous studies have shown that SHP-1 mRNA and protein are markedly decreased in aggressive lymphomas, and reduced SHP-1 levels are involved in the evolution of undetermined significance into multiple myeloma (34-36). DNA methylation is the most common mechanism of SHP-1 gene silencing in several types of hematopoietic tumors $(5,15,16,35,36)$. Therefore, we analyzed the frequency of SHP-1 DNA methylation in cells from patients in different phases of CML and in K562 cells by MSP and UMSP. We detected SHP-1 methylation in 23.8\% (10/42) of CP-CML cells, all of the AP-CML, BP-CML and K562 cells and none of the healthy donor cells, indicating 
that SHP-1 promoter methylation correlates with the reduced expression of SHP-1 observed during CML progression.

To further investigate the potential mechanism by which the SHP-1 gene functions in the progression to blast crisis in CML, we stably expressed SHP-1 in the K562 cell line and showed that transfection of SHP-1 into K562 cells could result in growth suppression, enhanced apoptosis and accumulation of cells in the G0/G1 phase. p210 ${ }^{\mathrm{BCR}-\mathrm{ABL} 1}$ transforms hematopoietic progenitor cells and plays a key role in the progression of CML, although specific PTPs that antagonize constitutively active BCR-ABL1 have not been sufficiently investigated. SHP-1 has been shown to be physically associated with BCR-ABL1 $(19,20)$, and our results demonstrated that forced expression of SHP-1 slightly decreased the level of $\mathrm{p} 210^{\mathrm{BCR}}$ ${ }^{\mathrm{ABL} 1}$ protein in $\mathrm{K} 562^{\mathrm{SHP}-1}$ cells; however, no changes were observed for BCR-ABL1 mRNA. We also showed that the overexpression of SHP-1 inhibited the tyrosine kinase activity of BCR-ABL1, according to the levels of pCrkL. Thus, our results indicate that SHP-1 suppresses oncogenic BCR-ABL1 kinase activity without influencing its transcription. A previous study indicated that PP2A, another phosphatase and tumor suppressor, makes BCR-ABL1 prone to proteasome-dependent proteolysis (37). Therefore, we propose that SHP-1 may also promote the degradation of BCR-ABL1 via the proteasome to decrease the level of $\mathrm{p} 210^{\mathrm{BCR}-\mathrm{ABL} 1}$.

The tumor-suppressing activity of SHP-1 depends on its ability to interact with and dephosphorylate several molecules implicated in cell cycle progression, proliferation, survival and differentiation (38). Remarkably, several targets are shared by the BCR-ABL1 kinase and the SHP-1 phosphatase. Among these, the expression and/or activity of the SHP-1 substrates JAK/STAT, RAS/MAPK, PI3K/AKT and MYC are either essential for BCR-ABL1 leukemogenesis or have been found to be altered in CML progression $(15,22,28,30-32,36,38,39)$. Our findings showed that forced expression of SHP-1 in K562 cells led to the inhibition of JAK2, STAT5, MAPK and AKT phosphorylation and decreased MYC expression. Moreover, by treating $\mathrm{K} 562^{\mathrm{SHP}-1}$ and $\mathrm{K} 562^{\mathrm{EGFP}}$ cells with $50 \mathrm{nM}$ IM (far lower than the $\mathrm{IC}_{50}$ concentration), we provide evidence that in CML, SHP-1 likely downregulates JAK2/STAT5, RAS/MAPK and PI3K/AKT signaling independently of BCR-ABL1 during disease progression.

In conclusion, our data demonstrate a novel BCR-ABL1independent mechanism of CML progression and reveal the correlation between SHP-1 expression and different phases of CML. SHP-1 most likely plays an important role in modulating the activation state of BCR-ABL1-dependent and -independent pathways as well as CML blastic transformation. Thus, the analysis of SHP-1 levels and the methylation status may be useful as an indicator of CML progression, although our results should be validated in a larger cohort of patients. Together, our findings suggest that SHP-1 may be used for the early assessment of blastic transformation in patients with CML and may also better tailor targeted therapy.

\section{Acknowledgements}

The authors thank Novartis Pharma AG for providing imatinib and nilotinib. The present study was supported by the Industry
Special Research Fund of the Health Ministry of China (no. 201202017).

\section{References}

1. Shtivelman E, Lifshitz B, Gale R and Canaani E: Fused transcript of abl and bcr genes in chronic myelogenous leukaemia. Nature 315: 550-554, 1985.

2. Ben-Neriah Y, Daley G, Mes-Masson A, Witte O and Baltimore D: The chronic myelogenous leukemia-specific P210 protein is the product of the BCR/ABL hybrid gene. Science 233: 212-214, 1986.

3. Quintas-Cardama A and Cortes J: Molecular biology of bcr-abllpositive chronic myeloid leukemia. Blood 113: 1619-1630, 2009.

4. Baccarani M, Deininger MW, Rosti G, et al: European LeukemiaNet recommendations for the management of chronic myeloid leukemia: 2013. Blood 122: 872-884, 2013.

5. Nash I: Chronic myeloid leukemia. N Engl J Med 341: 765, 1999.

6. Perrotti D, Jamieson C, Goldman J and Skorski T: Chronic myeloid leukemia: mechanisms of blastic transformation. J Clin Invest 120: 2254-2264, 2010.

7. Chen Y, Peng C, Li D and Li S: Molecular and cellular bases of chronic myeloid leukemia. Protein Cell 1: 124-132, 2010.

8. Rumpold $\mathrm{H}$ and Webersinke G: Molecular pathogenesis of Philadelphia-positive chronic myeloid leukemia - is it all BCR-ABL? Curr Cancer Drug Targets 11: 3-19, 2011.

9. Radich JP, Dai H, Mao M, et al: Gene expression changes associated with progression and response in chronic myeloid leukemia. Proc Natl Acad Sci USA 103: 2794-2799, 2006.

10. Feng $\mathrm{G}$, Hui $\mathrm{C}$ and Pawson $\mathrm{T}$ : $\mathrm{SH} 2$-containing phosphotyrosine phosphatase as a target of protein-tyrosine kinases. Science 259: 1607-1611, 1993.

11. Lorenz U: SHP-1 and SHP-2 in T cells: two phosphatases functioning at many levels. Immunol Rev 228: 342-359, 2009.

12. Li J, Xue L, Hao H, Han Y, Yang J and Luo J: Rapamycin provides a therapeutic option through inhibition of mTOR signaling in chronic myelogenous leukemia. Oncol Rep 27: 461-466, 2012.

13. Dong Q, Siminovitch KA, Fialkow L, Fukushima T and Downey GP: Negative regulation of myeloid cell proliferation and function by the $\mathrm{SH} 2$ domain-containing tyrosine phosphatase-1. J Immunol 162: 3220-3230, 1999.

14. Tapley P, Shevde N, Schweitzer P, et al: Increased G-CSF responsiveness of bone marrow cells from hematopoietic cell phosphatase deficient viable motheaten mice. Exp Hematol 25: 122-131, 1997.

15. Oka T, Ouchida M, Koyama M, et al: Gene silencing of the tyrosine phosphatase SHP1 gene by aberrant methylation in leukemias/lymphomas. Cancer Res 62: 6390-6394, 2002.

16. Chim CS, Fung TK, Cheung WC, Liang R and Kwong YL: SOCS1 and SHP1 hypermethylation in multiple myeloma: implications for epigenetic activation of the Jak/STAT pathway. Blood 103: 4630-4635, 2004

17. Zhang Q, Raghunath P, Vonderheid E, Odum N and Wasik M: Lack of phosphotyrosine phosphatase SHP-1 expression in malignant T-cell lymphoma cells results from methylation of the SHP-1 promoter. Am J Pathol 157: 1137-1146, 2000.

18. Esposito N, Colavita I, Quintarelli C, et al: SHP-1 expression accounts for resistance to imatinib treatment in Philadelphia chromosome-positive cells derived from patients with chronic myeloid leukemia. Blood 118: 3634-3644, 2011.

19. Bruecher-Encke B, Griffin J, Neel B and Lorenz U: Role of the tyrosine phosphatase SHP-1 in K562 cell differentiation. Leukemia 15: 1424-1432, 2001

20. Kharbanda S, Bharti A, Pei D, et al: The stress response to ionizing radiation involoves $\mathrm{c}$-Abl-dependent phosphorylation of SHPTP1. Proc Natl Acad Sci USA 93: 6898-6901, 1996.

21. Hamilton A, Elrick L, Myssina S, et al: BCR-ABL activity and its response to drugs can be determined in $\mathrm{CD} 34^{+} \mathrm{CML}$ stem cells by CrkL phosphorylation status using flow cytometry. Leukemia 20: $1035-1039,2006$

22. Lucas C, Harris R, Giannoudis A, Knight K, Watmough S and Clark R: BCR-ABL1 tyrosine kinase activity at diagnosis, as determined via the $\mathrm{pCrkL} / \mathrm{CrkL}$ ratio, is predictive of clinical outcome in chronic myeloid leukaemia. Br J Haematol 149: 458-460, 2010.

23. Siegel R, Naishadham D and Jemal A: Cancer statistics, 2012. CA Cancer J Clin 62: 10-29, 2012. 
24. Khoury H, Kukreja M, Goldman J, et al: Prognostic factors for outcomes in allogeneic transplantation for CML in the imatinib era: a CIBMTR analysis. Bone Marrow Transplant 47: 810-816, 2012.

25. Chong $\mathrm{Z}$ and Maiese $\mathrm{K}$ : The Src homology 2 domain tyrosine phosphatases SHP-1 and SHP-2: diversified control of cell growth, inflammation, and injury. Histol Histopathol 22: 1251-1267, 2007.

26. Rawlings JS, Rosler KM and Harrison DA: The JAK/STAT signaling pathway. J Cell Sci 117: 1281-1283, 2004.

27. Warsch W, Kollmann K, Eckelhart E, et al: High STAT5 levels mediate imatinib resistance and indicate disease progression in chronic myeloid leukemia. Blood 117: 3409-3420, 2011.

28. Xie S, Wang Y, Liu J, et al: Involvement of Jak2 tyrosine phosphorylation in Bcr-Abl transformation. Oncogene 20: 6188-6195, 2001 .

29. Samanta AK, Lin H, Sun T, Kantarjian H and Arlinghaus RB Janus kinase 2: a critical target in chronic myelogenous leukemia. Cancer Res 66: 6468-6472, 2006.

30. Samanta A, Chakraborty S, Wang Y, et al: Jak2 inhibition deactivates Lyn kinase through the SET-PP2A-SHP1 pathway, causing apoptosis in drug-resistant cells from chronic myelogenous leukemia patients. Oncogene 28: 1669-1681, 2009.

31. Samanta A, Perazzona B, Chakraborty S, et al: Janus kinase 2 regulates Bcr-Abl signaling in chronic myeloid leukemia. Leukemia 25: 463-472, 2011.

32. Luo J, Liu Z, Hao H, Wang F, Dong Z and Ryuzo O: Mutation analysis of hematopoietic cell phosphatase gene in acute leukemia. Zhongguo Shi Yan Xue Ye Xue Za Zhi 12: 128-132, 2004 (In Chinese).
33. Amin H, Hoshino K, Yang H, Lin Q, Lai R and Garcia-Manero G: Decreased expression level of SH2 domain-containing protein tyrosine phosphatase-1 (Shp1) is associated with progression of chronic myeloid leukaemia. J Pathol 212: 402-410, 2007.

34. Chim CS, Liang R, Leung MH and Kwong YL: Aberrant gene methylation implicated in the progression of monoclonal gammopathy of undetermined significance to multiple myeloma. J Clin Pathol 60: 104-106, 2007.

35. Zhang Q, Wang HY, Marzec M, Raghunath PN, Nagasawa T and Wasik MA: STAT3- and DNA methyltransferase 1-mediated epigenetic silencing of SHP-1 tyrosine phosphatase tumor suppressor gene in malignant T lymphocytes. Proc Natl Acad Sci USA 102: 6948-6953, 2005 .

36. Han Y, Amin HM, Franko B, Frantz C, Shi X and Lai R: Loss of SHP1 enhances JAK3/STAT3 signaling and decreases proteosome degradation of JAK3 and NPM-ALK in $\mathrm{ALK}^{+}$anaplastic large-cell lymphoma. Blood 108: 2796-2803, 2006.

37. Neviani P, Santhanam R, Trotta R, et al: The tumor suppressor PP2A is functionally inactivated in blast crisis CML through the inhibitory activity of the BCR/ABL-regulated SET protein Cancer Cell 8: 355-368, 2005.

38. Wu C, Sun M, Liu L and Zhou G: The function of the protein tyrosine phosphatase SHP-1 in cancer. Gene 306: 1-12, 2003.

39. Radich JP: The biology of CML blast crisis. Hematology 2007: 384-391, 2007. 